Received: 7 July 2017

Accepted: 5 October 2017

Published online: 19 October 2017

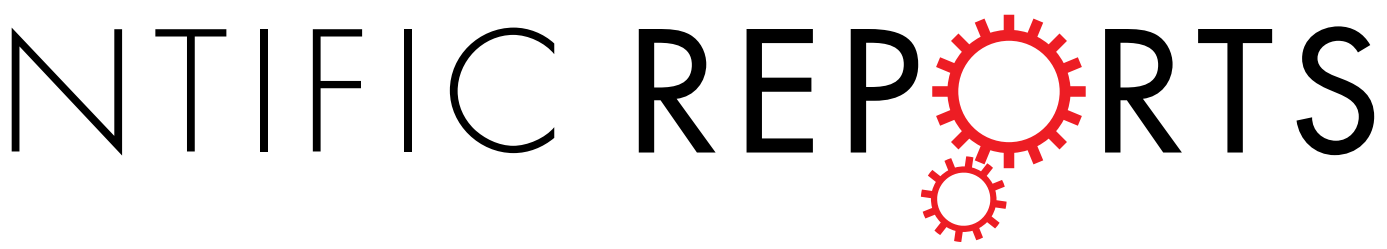

\title{
Associations of Blood Pressure with the Factors among Adults in Jilin Province: A Cross-Sectional Study Using Quantile Regression Analysis
}

Junsen Ye, Zhongmin Li, Yaogai Lv, Lan An, Jianxing Yu, Xin Guo, Yan Yao, Yaqin Yu \& Lina Jin

Hypertension has become a major public health challenge. However, numerous research results reported in the literature focus primarily on risk factors of hypertension, little is known about how the whole continuum of blood pressure (BP) is associated with risk factors of hypertension. This study aims to reveal quantile-specific associations of $B P$ with its risk factors. A cross-sectional survey based on a sample of 23,050 adults aged 18 to 79 years was conducted in Jilin Province in 2012, and some subjects were excluded due to missing values in BP or having BP control according to the purpose of this study. Quantile regression (QR) was employed to investigate the associations between systolic/diastolic blood pressure (SBP/DBP) and the risk factors. The SBP and DBP in males presented statistically higher than females $(P<0.001)$. High-salt diet for males manifested a slightly increasing positive association with higher SBP only for high quantiles ( $\geq 70$ ), but with a higher DBP for middle part of the quantiles (30 75), compared with bland diet. High-salt diet, drinking and high-density lipoprotein cholesterol (HDL-C) were positively associated with BP measures in males. And the coefficient of total cholesterol (TC) in QR increased with BP in females who used to live in town.

Hypertension is believed as a significant risk factor for cardiovascular disease (CVD), and has brought heavy economic burden to individuals, families, and society ${ }^{1-4}$. It is a great challenge for public health worldwide, because of its high prevalence, which accounts for nearly half of the cardiovascular morbidity and mortality in the world ${ }^{5,6}$. In 2010, people died from hypertension approximately 2 million in China, accounting for about $24.60 \%$ of total mortality ${ }^{7}$. Therefore, hypertension is an important public health problem that are supposed to be addressed urgently.

At present, hypertension has become a hot research $\operatorname{spot}^{8,9}$, where most of studies focused primarily on hypertension, including prevalence, influencing factors, and health problems in cross-sectional and cohort studies ${ }^{10-13}$. Sun et al's findings suggested that waist circumference (WC) was an independent predictor of hypertension incidence $^{10}$, and Zhang et al's prospective study suggested that body mass index (BMI) dynamic gain may be related to incident hypertension for men of all ages and young and middle-aged women ${ }^{13}$. Generally, hypertension was viewed as a categorical variable in these studies, but the incidence of hypertension is a chronic and continuous process. In other words, literatures about risk factors of hypertension have been mounting, yet there is a little of publications on determinants of the normal parts of blood pressure (BP).

Fortunately, quantile regression $(\mathrm{QR})$ is not limited to explain the prevalence risk of hypertension. What's more, it could also be used to explain the risk of the BP at any point on its distribution. QR comprehensively shows heterogeneous changes in the dispersion of the relationship of risk factors with BP continuum across its distribution. In addition, there is no distributional assumptions about the error term in QR model, thus, it enjoys high flexibility for modeling data with heterogeneous conditional distributions ${ }^{14}$.

In this study, we aimed mainly to investigate how the whole continuum of BP was associated with commonly researched influencing factors of hypertension using QR model. Jilin Province is situated in the northeast of China (latitude $40^{\circ} \sim 46^{\circ}$, longitude $121^{\circ} \sim 131^{\circ}$ ), with a cold climate and different residential dietary pattern ${ }^{1,15}$. Therefore, we could develop more effective prevention strategies for different BP populations, so as to achieve the purpose of "precision prevention". 


\begin{tabular}{|c|c|c|c|c|c|}
\hline Variable & Total $(n=16524)$ & Male $(\mathrm{n}=7607)$ & Female $(n=8917)$ & $t / \chi^{2}$ & $P$ value \\
\hline Age (year) & $47.79 \pm 13.16$ & $47.02 \pm 13.73$ & $48.45 \pm 12.63$ & 0.6893 & $<0.001$ \\
\hline BMI $\left(\mathrm{kg} / \mathrm{m}^{2}\right)$ & $24.26 \pm 3.66$ & $24.34 \pm 3.66$ & $24.19 \pm 3.66$ & 2.555 & 0.011 \\
\hline SBP (mmHg) & $131.33 \pm 21.33$ & $134.46 \pm 19.74$ & $128.67 \pm 22.25$ & 17.750 & $<0.001$ \\
\hline DBP (mmHg) & $79.99 \pm 11.73$ & $82.34 \pm 11.71$ & $77.99 \pm 11.38$ & 24.095 & $<0.001$ \\
\hline $\mathrm{TG}(\mathrm{mmol} / \mathrm{L})$ & $1.96 \pm 1.80$ & $2.17 \pm 2.09$ & $1.79 \pm 1.49$ & 13.508 & $<0.001$ \\
\hline $\mathrm{TC}(\mathrm{mmol} / \mathrm{L})$ & $4.90 \pm 1.08$ & $4.88 \pm 1.06$ & $4.92 \pm 1.10$ & -2.154 & 0.031 \\
\hline LDL-C (mmol/L) & $2.94 \pm 0.89$ & $2.89 \pm 0.86$ & $2.97 \pm 0.91$ & -5.690 & $<0.001$ \\
\hline HDL-C $(\mathrm{mmol} / \mathrm{L})$ & $1.39 \pm 0.39$ & $1.35 \pm 0.41$ & $1.42 \pm 0.36$ & -11.936 & $<0.001$ \\
\hline FPG (mmol/L) & $5.39 \pm 1.66$ & $5.53 \pm 1.68$ & $5.27 \pm 1.64$ & 9.936 & $<0.001$ \\
\hline \multicolumn{6}{|l|}{ Education } \\
\hline Compulsory & $4985(30.2)$ & $1696(22.3)$ & $3289(36.9)$ & 422.429 & $<0.001$ \\
\hline High school & $8981(54.4)$ & 4539(59.7) & $4442(49.8)$ & & \\
\hline Undergraduate & $2558(15.5)$ & 1372(18.0) & $1186(13.3)$ & & \\
\hline \multicolumn{6}{|l|}{ Occupation } \\
\hline Other* & $4018(24.3)$ & $1218(16.0)$ & $2800(31.4)$ & 621.136 & $<0.001$ \\
\hline Manual labor & $7914(47.9)$ & $4302(56.6)$ & $3612(40.5)$ & & \\
\hline Mental labor & $4592(27.8)$ & 2087(27.4) & $2505(28.1)$ & & \\
\hline \multicolumn{6}{|l|}{ Residence } \\
\hline Rural & $8484(51.3)$ & $3959(52.0)$ & $4525(50.7)$ & 2.770 & 0.050 \\
\hline Town & $8040(48.7)$ & $3648(48.0)$ & 4392(49.3) & & \\
\hline \multicolumn{6}{|l|}{ Drinking } \\
\hline No & $11393(68.9)$ & $3302(43.4)$ & 8091(90.7) & 4295.061 & $<0.001$ \\
\hline Yes & $5131(31.1)$ & 4305(56.6) & $826(9.3)$ & & \\
\hline \multicolumn{6}{|l|}{ Smoking } \\
\hline No & $11512(67.9)$ & $3634(47.8)$ & $7878(88.3)$ & 3198.384 & $<0.001$ \\
\hline Yes & $5012(30.3)$ & $3973(52.2)$ & $1039((11.7)$ & & \\
\hline \multicolumn{6}{|l|}{ high - salt diet } \\
\hline No & $10242(62.0)$ & $4229(55.6)$ & 6013(67.4) & 244.190 & $<0.001$ \\
\hline Yes & $6282(38.0)$ & $3378(44.4)$ & $2904(32.6)$ & & \\
\hline \multicolumn{6}{|c|}{ Family history of CVD } \\
\hline No & $8456(51.2)$ & $4158(54.7)$ & $4298(48.2)$ & 68.564 & $<0.001$ \\
\hline Yes & $8068(48.8)$ & $3449(45.3)$ & 4619(51.8) & & \\
\hline \multicolumn{6}{|l|}{ Hypertension } \\
\hline No & $10368(62.7)$ & $4479(58.9)$ & $5589(66.0)$ & 90.089 & $<0.001$ \\
\hline Yes & $6165(37.3)$ & $3128(41.1)$ & $3028(34.0)$ & & \\
\hline \multicolumn{6}{|l|}{ Diabetes } \\
\hline No & $9942(60.2)$ & $4229(55.6)$ & $5713(64.1)$ & 123.028 & $<0.001$ \\
\hline Yes & $6582(39.8)$ & $3378(44.4)$ & $3204(35.9)$ & & \\
\hline \multicolumn{6}{|l|}{ Dyslipidemia } \\
\hline No & $14855(89.9)$ & 6793(89.3) & $8062(90.4)$ & 5.477 & 0.019 \\
\hline Yes & $1668(10.1)$ & $813(10.7)$ & $855(9.6)$ & & \\
\hline
\end{tabular}

Table 1. Descriptive characteristics of participants by gender $[\bar{X} \pm S, \mathrm{n}(\%)]$. * "other" included unemployed and retired people.

\section{Results}

Descriptive characteristics of participants by gender. Table 1 shows the basic characteristics of the participants, where BMI, SBP, DBP, TG and FPG were all significantly higher in males than those in females $(P<0.05)$, but age, TC, LDL-C and HDL-C were just the opposite $(P<0.05)$. And the proportions of demographics (drinking, smoking, hypertension, dyslipidemia and diabetes, etc.) were significantly different between genders. As shown in Table 2, the distribution of BP and quantiles between males and females were different. Therefore, we separately identified the factors for males and females by using QR model.

QR statistics between the BP measures in females. Tables 3 4 show QR coefficients and 95\% confidence intervals of the influencing factors for BP in females. Age was positively associated with both SBP and DBP. Similarly, BMI, TG, TC and family history of CVD showed significantly positive associations with SBP/DBP across the entire conditional BP distribution. Females who used to live in town were positively associated with a higher DBP/SBP for most of the quantiles $(\geq 10)$. Undergraduate females were negatively associated with SBP for most of the quantiles $(\geq 20)$. Middle school females were negatively associated with SBP for part of the quantiles. 


\begin{tabular}{|c|c|c|c|c|c|c|c|c|c|c|c|c|}
\hline \multirow[b]{2}{*}{ Variable } & \multicolumn{12}{|c|}{ Quantiles } \\
\hline & 10 & 20 & 30 & 40 & 50 & 60 & 70 & 75 & 80 & 85 & 90 & 95 \\
\hline \multicolumn{13}{|l|}{ Male } \\
\hline SBP(mmHg) & 113 & 118 & 122 & 126 & 130 & 135 & 140 & 144 & 149 & 153 & 160 & 173 \\
\hline $\mathrm{DBP}(\mathrm{mmHg})$ & \begin{tabular}{|l|}
68 \\
\end{tabular} & 72 & 75 & 78 & 80 & 84 & 87 & 89 & 91 & 94 & 97 & 102 \\
\hline \multicolumn{13}{|l|}{ Female } \\
\hline $\mathrm{SBP}(\mathrm{mmHg})$ & \begin{tabular}{|l|}
104 \\
\end{tabular} & 110 & 114 & 119 & 124 & 129 & 136 & 140 & 145 & 152 & 159 & 171 \\
\hline $\mathrm{DBP}(\mathrm{mmHg})$ & 64 & 68 & 71 & 74 & 77 & 79 & 82 & 84 & 86 & 89 & 92 & 98 \\
\hline
\end{tabular}

Table 2. Quantiles of blood pressure (SBP \& DBP) by gender. Numbers in the Table are blood pressure at different quantiles.

Female manual labors and mental labors were positively associated with a higher DBP for part of the quantiles, compared with unemployed and retired females.

QR statistics between the BP measures in males. Tables $5 \sim 6$ provide QR coefficients and $95 \%$ confidence intervals of the influencing factors for BP in males. Similar to females, age, BMI, TG and family history of CVD presented positive associations with both BP measures across the entire conditional BP distribution. Drinking showed positive associations with SBP/DBP. High-salt diet manifested a slightly increasing positive association with higher SBP (quantiles $\geq 70$ ), but with a higher DBP for middle part of the quantiles (30 75), compared with the bland diet. Undergraduate males were negatively associated with SBP for most of the quantiles $(\geq 20)$. Male manual labors and mental labors were positively associated with a higher DBP across the entire conditional DBP distribution compared with unemployed and retired males.

In addition, HDL-C also showed a positive association with SBP/DBP. In general, the quantiles of HDL-C among participants with hypertension were lower than those with normal BP in males (Table 7). In contrast, the relationship changed in different BMI groups: the relationship remained the same in normal weight group, however, the quantiles of HDL-C among participants with hypertension were higher than those with normal BP in underweight, overweight and obese groups. Further, the results of generalized additive models (GAM) showed that HDL-C performed non-linear associations with both $\operatorname{SBP}(F=3.513, P=0.012)$ and $\mathrm{DBP}(F=7.388$, $P<0.001$ ) (Fig. 1).

\section{Discussion}

Hypertension has become a major public health problem in $\mathrm{China}^{7}$, and a number of researchers have investigated the risk factors of hypertension ${ }^{5,16}$, but little is known about how the whole continuum of DBP and SBP is associated with commonly researched influencing factors of hypertension. Measures in reducing the prevalence of hypertension in Jilin Province could be put forward after knowing the risk factors associated with BP. This study explored the heterogeneity of the relationship of environmental and individual determinants with hypertension across the entire conditional distribution of BP measures using a QR model.

It was indicated that unhealthy eating habits was a predictor for higher BP measures in males, with a larger extent of the positive association at a higher quantile. Previous studies have reported that BP was more prone to be elevated by alcohol drinking in males than in females ${ }^{17}$. In addition, it has been proven that high-salt diet was associated with $\mathrm{BP}^{18}$. Our results showed that the high-salt was positively associated with $\mathrm{BP}$ only for the males, and the mechanism was probably correlated with estrogen and salt sensitivity. Estrogen affects the sodium reabsorption and excretion via up regulation of $\mathrm{NO}$ production and different expression level of angiotensin receptors and ET receptors ${ }^{19}$. Therefore, males who maintain healthy lifestyle are also likely to manage their BP better, and thereby have a lower risk of hypertension.

Our study also investigated the effects of BMI and serum lipids on BP among adults. BMI showed a positive association with BP in our study, which was consistent with the literature that obese people were more likely to be hypertensive compared with nonobese people ${ }^{20}$. Activation of the renin-angiotensin system as well as physical compression of the kidney might be important factors in linking body weight and elevated $\mathrm{BP}^{21}$. Further, it has been proven that dyslipidemia was associated with $\mathrm{BP}^{22}$. Our results showed that TG level was positively correlated with $\mathrm{BP}$, and the mechanism was probably related with the insulin resistance ${ }^{23}$. Elevated TG level could make a higher level of free fatty acids in serum, which would lead to insulin resistance, then insulin resistance further contributed to hyperinsulinemia, which might directly contribute to elevation of BP by increasing renal sodium retention ${ }^{24}$. However, TC and HDL-C showed different associations with BP between males and females, which might be attributed to differences in sex hormones, where estrogen was commonly believed to play an important role in lipolysis ${ }^{25}$. Moreover, HDL-C was positively associated with SBP/DBP in males, which was inconsistent with the results in literature ${ }^{21,26}$. One possible reason was the nonlinear relationship between HDL-C and SBP/DBP, which showed a " $U$ " shape that both too lower and too higher levels of HDL-C might increase the risk of hypertension. Further, there might be an interaction between BMI and HDL-C, which showed that the HDL-C was higher among participants with hypertension than those with normal BP in almost all BMI groups except those with normal BMI. Besides, other confounding risk factors ${ }^{27,28}$ that were not under our consideration might also have impacts on this association.

Furthermore, it has been proven that education was associated with hypertension ${ }^{29}$. Our results showed that education negatively associated with SBP. On one hand, the people with higher level of education would have greater possibilities to engage in healthy lifestyles. On the other hand, older people were expected to have lower 


\begin{tabular}{|c|c|c|c|c|c|c|c|c|c|c|c|c|}
\hline & & \multicolumn{11}{|l|}{ Quantile } \\
\hline \multicolumn{2}{|l|}{ Variables } & 10 & 20 & 30 & 40 & 50 & 60 & 70 & 75 & 80 & 85 & 90 \\
\hline Age (year) & & $\begin{array}{l}0.33^{*} \\
{[0.29,0.36]}\end{array}$ & $\begin{array}{l}0.43^{*} \\
{[0.39,0.46]}\end{array}$ & \begin{tabular}{|l|}
$0.50^{*}$ \\
{$[0.47,0.53]$}
\end{tabular} & \begin{tabular}{|l}
$0.59 *$ \\
{$[0.55,0.62]$}
\end{tabular} & \begin{tabular}{|l|}
$0.67^{*}$ \\
{$[0.63,0.70]$}
\end{tabular} & \begin{tabular}{|l|}
$0.75^{*}$ \\
{$[0.72,0.79]$}
\end{tabular} & \begin{tabular}{|l|}
$0.82 *$ \\
{$[0.78,0.86]$}
\end{tabular} & \begin{tabular}{|l|}
$0.88^{*}$ \\
{$[0.84,0.93]$}
\end{tabular} & $\begin{array}{l}0.94^{*} \\
{[0.89,0.98]}\end{array}$ & $\begin{array}{l}1.02 * \\
{[0.96,1.08]}\end{array}$ & \begin{tabular}{|l}
$1.07^{*}$ \\
{$[1.01,1.15]$}
\end{tabular} \\
\hline $\begin{array}{l}\mathrm{BMI}(\mathrm{Kg} / \\
\left.\mathrm{m}^{2}\right)\end{array}$ & & \begin{tabular}{|l|}
$1.00 *$ \\
{$[0.90,1.09]$}
\end{tabular} & $\begin{array}{l}0.93 * \\
{[0.84,1.05]}\end{array}$ & \begin{tabular}{|l}
$1.06^{*}$ \\
{$[0.96,1.16]$}
\end{tabular} & $\begin{array}{l}1.09 * \\
{[0.99,1.23]}\end{array}$ & \begin{tabular}{|l|}
$1.22 *$ \\
{$[1.11,1.31]$}
\end{tabular} & \begin{tabular}{|l}
$1.23 *$ \\
{$[1.12,1.35]$}
\end{tabular} & \begin{tabular}{|l}
$1.28^{*}$ \\
{$[1.15,1.42]$}
\end{tabular} & \begin{tabular}{|l|}
$1.28^{*}$ \\
{$[1.13,1.44]$}
\end{tabular} & $\begin{array}{l}1.32^{*} \\
{[1.15,1.51]}\end{array}$ & $\begin{array}{l}1.48^{*} \\
{[1.24,1.67]}\end{array}$ & \begin{tabular}{|l|}
$1.63 *$ \\
{$[1.41,1.85]$}
\end{tabular} \\
\hline \multirow[b]{2}{*}{ Residence } & Rural & - & - & - & - & - & - & - & - & - & - & - \\
\hline & Town & $\begin{array}{l}0.30 \\
{[-0.42,1.03]}\end{array}$ & $\begin{array}{l}0.79^{*} \\
{[0.29,1.78]}\end{array}$ & $\begin{array}{l}1.57^{*} \\
{[0.84,2.14]}\end{array}$ & $\begin{array}{l}1.69^{*} \\
{[0.92,2.40]}\end{array}$ & $\begin{array}{l}1.76^{*} \\
{[1.02,2.42]}\end{array}$ & $\begin{array}{l}2.12^{*} \\
{[1.18,2.79]}\end{array}$ & $\begin{array}{l}3.22 * \\
{[2.35,4.01]}\end{array}$ & $\begin{array}{l}3.40^{*} \\
{[2.21,4.61]}\end{array}$ & $\begin{array}{l}3.15^{*} \\
{[2.18,4.35]}\end{array}$ & $\begin{array}{l}3.16^{*} \\
{[1.92,4.89]}\end{array}$ & $\begin{array}{l}4.22^{*} \\
{[2.78,5.63]}\end{array}$ \\
\hline \multirow{2}{*}{$\begin{array}{l}\text { Family } \\
\text { history of } \\
\text { CVD }\end{array}$} & No & - & - & - & - & - & - & - & - & - & - & - \\
\hline & Yes & \begin{tabular}{|l|}
$1.97 *$ \\
{$[1.34,2.64]$}
\end{tabular} & \begin{tabular}{|l}
$2.24 *$ \\
{$[1.54,2.87]$}
\end{tabular} & \begin{tabular}{|l|}
$2.76^{*}$ \\
{$[2.12,3.37]$}
\end{tabular} & \begin{tabular}{|l|}
$2.92^{*}$ \\
{$[2.25,3.55]$}
\end{tabular} & \begin{tabular}{|l|}
$2.90^{*}$ \\
{$[2.25,3.56]$}
\end{tabular} & \begin{tabular}{|l|}
$2.96^{*}$ \\
{$[2.33,3.70]$}
\end{tabular} & \begin{tabular}{|l|}
$2.74^{*}$ \\
{$[1.86,3.54]$}
\end{tabular} & \begin{tabular}{|l}
$3.14^{*}$ \\
{$[2.02,4.08]$}
\end{tabular} & $\begin{array}{l}3.43^{*} \\
{[2.53,4.36]}\end{array}$ & $\begin{array}{l}4.14^{*} \\
{[2.98,5.34]}\end{array}$ & \begin{tabular}{|l|}
$4.89^{*}$ \\
{$[3.22,5.94]$}
\end{tabular} \\
\hline \multirow{3}{*}{ Education } & Compulsory & - & - & - & - & - & - & - & - & - & - & - \\
\hline & High school & $\begin{array}{l}-0.71 \\
{[-1.52,0.17]}\end{array}$ & $\begin{array}{l}-0.83 \\
{[-1.54,0.24]}\end{array}$ & $\begin{array}{l}-0.57 \\
{[-1.47,0.27]}\end{array}$ & $\begin{array}{l}-0.81^{*} \\
{[-1.61,-0.03]}\end{array}$ & $\begin{array}{l}-1.06^{*} \\
{[-1.76,-0.31]}\end{array}$ & \begin{tabular}{|l|}
$-0.94^{*}$ \\
{$[-2.09,-0.12]$}
\end{tabular} & $\begin{array}{l}-0.90 \\
{[-2.09,0.2]}\end{array}$ & \begin{tabular}{|l|}
-0.75 \\
{$[-2.31,0.46]$}
\end{tabular} & $\begin{array}{l}-1.21^{*} \\
{[-2.31,-0.02]}\end{array}$ & \begin{tabular}{|l|}
-1.68 \\
{$[-3.41,0.23]$}
\end{tabular} & \begin{tabular}{|l|}
-1.81 \\
{$[-4.34,0.28]$}
\end{tabular} \\
\hline & Undergraduate & $\begin{array}{l}-0.96^{*} \\
{[-1.97,-0.15]}\end{array}$ & $\begin{array}{l}-1.90 * \\
{[-3.02,-0.69]}\end{array}$ & \begin{tabular}{|l|}
$-1.66^{*}$ \\
{$[-2.48,-0.61]$}
\end{tabular} & \begin{tabular}{|l|}
$-1.99^{*}$ \\
{$[-3.29,-0.89]$}
\end{tabular} & $\begin{array}{l}-2.12 * \\
{[-3.3,-1.01]}\end{array}$ & \begin{tabular}{|l|}
$-2.04^{*}$ \\
{$[-3.17,-0.92]$}
\end{tabular} & $\begin{array}{l}-2.63 * \\
{[-4.2,-1.36]}\end{array}$ & $\begin{array}{l}-3.06^{*} \\
{[-4.82,-1.34]}\end{array}$ & $\begin{array}{l}-3.96^{*} \\
{[-5.33,-1.89]}\end{array}$ & \begin{tabular}{|l|}
$-5.39 *$ \\
{$[-6.92,-3.05]$}
\end{tabular} & \begin{tabular}{|l|}
$-5.91^{*}$ \\
{$[-8.71,-3.26]$}
\end{tabular} \\
\hline \multirow{2}{*}{$\begin{array}{l}\text { Blood } \\
\text { lipid }\end{array}$} & TG & $\begin{array}{l}0.81 * \\
{[0.62,1.07]}\end{array}$ & \begin{tabular}{|l|}
$0.77^{*}$ \\
{$[0.36,1.01]$}
\end{tabular} & \begin{tabular}{|l|}
$0.81^{*}$ \\
{$[0.64,1.01]$}
\end{tabular} & \begin{tabular}{|l|}
$0.75^{*}$ \\
{$[0.47,1.01]$} \\
\end{tabular} & \begin{tabular}{|l|}
$0.87^{*}$ \\
{$[0.44,1.18]$}
\end{tabular} & \begin{tabular}{|l|}
$0.90^{*}$ \\
{$[0.61,1.16]$}
\end{tabular} & \begin{tabular}{|l|}
$1.18^{*}$ \\
{$[0.66,1.61]$}
\end{tabular} & \begin{tabular}{|l|}
$1.43^{*}$ \\
{$[0.81,1.99]$}
\end{tabular} & \begin{tabular}{|l|}
$1.59^{*}$ \\
{$[1.13,2.08]$}
\end{tabular} & \begin{tabular}{|l|}
$1.77^{*}$ \\
{$[1.13,2.37]$}
\end{tabular} & \begin{tabular}{|l|}
$1.89^{*}$ \\
{$[1.00,2.27]$}
\end{tabular} \\
\hline & TC & $\begin{array}{l}0.15 \\
{[-0.10,0.62]}\end{array}$ & $\begin{array}{l}0.53^{*} \\
{[0.21,0.86]}\end{array}$ & \begin{tabular}{|l|}
$0.54^{*}$ \\
{$[0.28,0.83]$}
\end{tabular} & \begin{tabular}{|l|}
$0.56^{*}$ \\
{$[0.13,0.97]$}
\end{tabular} & \begin{tabular}{|l}
$0.69^{*}$ \\
{$[0.33,1.06]$}
\end{tabular} & \begin{tabular}{|l|}
$0.89^{*}$ \\
{$[0.38,1.28]$}
\end{tabular} & $\begin{array}{l}1.12 * \\
{[0.55,1.53]}\end{array}$ & \begin{tabular}{|l|}
$0.91^{*}$ \\
{$[0.14,1.49]$}
\end{tabular} & $\begin{array}{l}0.79^{*} \\
{[0.28,1.31]}\end{array}$ & $\begin{array}{l}0.38 \\
{[-0.22,1.02]}\end{array}$ & $\begin{array}{l}0.30 \\
{[-0.40,1.29]}\end{array}$ \\
\hline
\end{tabular}

Table 3. Quantile regression coefficients [ $95 \%$ confidence intervals] of influencing factors for SBP in females. Numbers in the Table are coefficient estimates from the quantile regression with $95 \%$ confidence intervals shown in brackets. ${ }^{*} P<0.05$.

\begin{tabular}{|c|c|c|c|c|c|c|c|c|c|c|c|c|}
\hline \multirow{2}{*}{\multicolumn{2}{|c|}{ Variables }} & \multicolumn{11}{|l|}{ Quantile } \\
\hline & & \multirow{2}{*}{$\begin{array}{l}10 \\
0.04^{*} \\
{[0.02,0.07]}\end{array}$} & \multirow{2}{*}{$\begin{array}{l}20 \\
0.07^{*} \\
{[0.06,0.09]}\end{array}$} & \multirow{2}{*}{\begin{tabular}{|l|}
30 \\
$0.09^{*}$ \\
{$[0.07,0.11]$}
\end{tabular}} & \multirow{2}{*}{\begin{tabular}{|l|}
40 \\
$0.11 *$ \\
{$[0.09,0.12]$} \\
\end{tabular}} & \multirow{2}{*}{$\begin{array}{l}50 \\
0.12^{*} \\
{[0.1,0.14]}\end{array}$} & \multirow{2}{*}{$\begin{array}{l}60 \\
0.14^{*} \\
{[0.11,0.17]}\end{array}$} & \multirow{2}{*}{$\begin{array}{l}70 \\
0.15^{*} \\
{[0.13,0.18]}\end{array}$} & \multirow{2}{*}{\begin{tabular}{|l}
75 \\
$0.17^{*}$ \\
{$[0.14,0.20]$} \\
\end{tabular}} & \multirow{2}{*}{$\begin{array}{l}80 \\
0.19^{*} \\
{[0.16,0.22]}\end{array}$} & \multirow{2}{*}{\begin{tabular}{|l}
85 \\
$0.20^{*}$ \\
{$[0.17,0.23]$}
\end{tabular}} & \multirow{2}{*}{$\begin{array}{l}90 \\
0.23 * \\
{[0.18,0.26]}\end{array}$} \\
\hline Age (year) & & & & & & & & & & & & \\
\hline $\begin{array}{l}\mathrm{BMI}(\mathrm{Kg} / \\
\left.\mathrm{m}^{2}\right)\end{array}$ & & $\begin{array}{l}0.64^{*} \\
{[0.54,0.73]}\end{array}$ & $\begin{array}{l}0.68^{*} \\
{[0.63,0.73]}\end{array}$ & $\begin{array}{l}0.71^{*} \\
{[0.65,0.76]} \\
\end{array}$ & $\begin{array}{l}0.72 * \\
{[0.66,0.78]} \\
\end{array}$ & $\begin{array}{l}0.77^{*} \\
{[0.73,0.83]} \\
\end{array}$ & $\begin{array}{l}0.81^{*} \\
{[0.74,0.87]}\end{array}$ & $\begin{array}{l}0.82 * \\
{[0.74,0.89]} \\
\end{array}$ & $\begin{array}{l}0.86^{*} \\
{[0.75,0.93]} \\
\end{array}$ & $\begin{array}{l}0.85^{*} \\
{[0.77,0.93]}\end{array}$ & $\begin{array}{l}0.85^{*} \\
{[0.77,0.94]} \\
\end{array}$ & $\begin{array}{l}0.97^{*} \\
{[0.83,1.04]}\end{array}$ \\
\hline \multirow[b]{2}{*}{ Residence } & Rural & - & - & - & - & - & - & - & - & - & - & - \\
\hline & Town & $\begin{array}{l}0.37 \\
{[-0.18,1.21]}\end{array}$ & \begin{tabular}{|l}
$0.62 *$ \\
{$[0.18,1.08]$} \\
\end{tabular} & $\begin{array}{l}0.78^{*} \\
{[0.32,1.24]} \\
\end{array}$ & \begin{tabular}{|l|}
$0.99 *$ \\
{$[0.51,1.46]$} \\
\end{tabular} & \begin{tabular}{|l|}
$1.09 *$ \\
{$[0.82,1.53]$} \\
\end{tabular} & $\begin{array}{l}1.05^{*} \\
{[0.56,1.49]} \\
\end{array}$ & \begin{tabular}{|l|}
$0.94^{*}$ \\
{$[0.32,1.47]$} \\
\end{tabular} & \begin{tabular}{|l|}
$0.93^{*}$ \\
{$[0.41,1.52]$} \\
\end{tabular} & $\begin{array}{l}1.29^{*} \\
{[0.38,1.83]}\end{array}$ & \begin{tabular}{|l|}
$0.90^{*}$ \\
{$[0.19,1.65]$} \\
\end{tabular} & $\begin{array}{l}1.04^{*} \\
{[0.05,1.87]} \\
\end{array}$ \\
\hline \multirow{2}{*}{$\begin{array}{l}\text { Family } \\
\text { history of } \\
\text { CVD }\end{array}$} & No & - & - & - & - & - & - & - & - & - & - & - \\
\hline & Yes & $\begin{array}{l}1.40^{*} \\
{[0.82,1.91]} \\
\end{array}$ & $\begin{array}{l}1.32 * \\
{[0.95,1.67]} \\
\end{array}$ & $\begin{array}{l}1.49^{*} \\
{[1.14,1.90]} \\
\end{array}$ & \begin{tabular}{|l|}
$1.70 *$ \\
{$[1.29,2.10]$} \\
\end{tabular} & \begin{tabular}{|l|}
$1.51^{*}$ \\
{$[1.18,1.85]$} \\
\end{tabular} & $\begin{array}{l}1.84^{*} \\
{[1.45,2.28]} \\
\end{array}$ & \begin{tabular}{|l|}
$2.24^{*}$ \\
{$[1.72,2.78]$} \\
\end{tabular} & \begin{tabular}{|l|}
$2.39 *$ \\
{$[1.90,2.94]$} \\
\end{tabular} & $\begin{array}{l}2.68^{*} \\
{[2.07,3.24]} \\
\end{array}$ & \begin{tabular}{|l|}
$2.78^{*}$ \\
{$[2.08,3.41]$} \\
\end{tabular} & $\begin{array}{l}2.64 * \\
{[1.91,3.31]} \\
\end{array}$ \\
\hline \multirow{3}{*}{ Occupation } & Other* & - & - & - & - & - & - & - & - & - & - & - \\
\hline & \begin{tabular}{|l|} 
Manual \\
labor
\end{tabular} & $\begin{array}{l}0.59 \\
{[-0.30,1.46]}\end{array}$ & \begin{tabular}{|l|}
$0.75^{*}$ \\
{$[0.27,1.20]$}
\end{tabular} & $\begin{array}{l}0.63^{*} \\
{[0.05,1.20]}\end{array}$ & \begin{tabular}{|l|}
$0.6^{*}$ \\
{$[0.09,1.25]$}
\end{tabular} & $\begin{array}{l}0.68^{*} \\
{[0.16,1.06]}\end{array}$ & $\begin{array}{l}0.68^{*} \\
{[0.13,1.23]}\end{array}$ & $\begin{array}{l}1.00^{*} \\
{[0.33,1.65]}\end{array}$ & $\begin{array}{l}1.19 * \\
{[0.60,1.92]}\end{array}$ & $\begin{array}{l}1.18^{*} \\
{[0.58,2.13]}\end{array}$ & $\begin{array}{l}1.32 * \\
{[0.60,2.09]}\end{array}$ & $\begin{array}{l}1.37 * \\
{[0.24,2.43]}\end{array}$ \\
\hline & $\begin{array}{l}\text { Mental } \\
\text { labor }\end{array}$ & $\begin{array}{l}0.09 \\
{[-0.95,0.96]}\end{array}$ & \begin{tabular}{|l|}
0.42 \\
{$[-0.08,0.97]$}
\end{tabular} & \begin{tabular}{|l|}
0.46 \\
{$[0.02,1.12]$}
\end{tabular} & \begin{tabular}{|l|}
0.36 \\
{$[-0.15,1.01]$}
\end{tabular} & \begin{tabular}{|l|}
0.54 \\
{$[-0.07,1.12]$}
\end{tabular} & \begin{tabular}{|l|}
$0.70^{*}$ \\
{$[0.00,1.28]$}
\end{tabular} & $\begin{array}{l}0.99^{*} \\
{[0.18,1.61]}\end{array}$ & $\begin{array}{l}0.99 * \\
{[0.42,1.83]}\end{array}$ & $\begin{array}{l}1.20^{*} \\
{[0.32,2.04]}\end{array}$ & $\begin{array}{l}0.73 \\
{[-0.25,1.58]}\end{array}$ & $\begin{array}{l}0.50 \\
{[-0.88,1.42]}\end{array}$ \\
\hline \multirow[t]{2}{*}{ Blood lipid } & TG & $\begin{array}{l}0.35^{*} \\
{[0.22,0.53]}\end{array}$ & \begin{tabular}{|l|}
$0.40^{*}$ \\
{$[0.24,0.61]$}
\end{tabular} & \begin{tabular}{|l|}
$0.62 *$ \\
{$[0.42,0.87]$}
\end{tabular} & \begin{tabular}{|l|}
$0.71^{*}$ \\
{$[0.52,0.82]$}
\end{tabular} & \begin{tabular}{|l|}
$0.62^{*}$ \\
{$[0.46,0.76]$}
\end{tabular} & $\begin{array}{l}0.67^{*} \\
{[0.4,0.89]}\end{array}$ & $\begin{array}{l}0.72^{*} \\
{[0.48,0.99]}\end{array}$ & $\begin{array}{l}0.75^{*} \\
{[0.54,1.00]}\end{array}$ & $\begin{array}{l}0.80^{*} \\
{[0.45,1.03]}\end{array}$ & $\begin{array}{l}0.68^{*} \\
{[0.47,1.14]}\end{array}$ & \begin{tabular}{|l|}
$0.78^{*}$ \\
{$[0.48,1.10]$}
\end{tabular} \\
\hline & $\mathrm{TC}$ & $\begin{array}{l}0.72^{*} \\
{[0.34,0.96]}\end{array}$ & $\begin{array}{l}0.57 * \\
{[0.32,0.78]}\end{array}$ & $\begin{array}{l}0.47 * \\
{[0.27,0.70]}\end{array}$ & $\begin{array}{l}0.53^{*} \\
{[0.36,0.73]}\end{array}$ & $\begin{array}{l}0.60^{*} \\
{[0.43,0.79]}\end{array}$ & $\begin{array}{l}0.51^{*} \\
{[0.26,0.75]}\end{array}$ & $\begin{array}{l}0.59 * \\
{[0.36,0.94]}\end{array}$ & $\begin{array}{l}0.69^{*} \\
{[0.42,1.03]}\end{array}$ & $\begin{array}{l}0.72 * \\
{[0.33,1.05]}\end{array}$ & $\begin{array}{l}0.86^{*} \\
{[0.42,1.28]}\end{array}$ & $\begin{array}{l}0.73 * \\
{[0.26,1.29]}\end{array}$ \\
\hline
\end{tabular}

Table 4. Quantile regression coefficients [ $95 \%$ confidence intervals] of influencing factors for DBP in females. Numbers in the Table are coefficient estimates from the quantile regression with $95 \%$ confidence intervals shown in brackets. ${ }^{*} P<0.05$, "other" included unemployed and retired people.

level of education than young people, which might also lead to higher $\mathrm{SBP}^{30}$. In addition, we also found that working people were more likely to have an elevated DBP, and the possible reason might be related to social engagement. Finally, the regression coefficient of family history of CVD increased with BP among all the research participates, which was consistent with other studies ${ }^{31}$. Thus, it was implied that elderly people with family history of CVD, low-literacy and workers should pay more attention to their BP levels.

Some limitations of our study should be noted. Firstly, the results were conducted from a cross-sectional study in Jilin province, which might limit our ability to generalize the results. Secondly, other confounders that might have implications for hypertension, such as parameters of glucose metabolism, renal function indices and genes, were not under our consideration this time, which might have some slight effects on our results.

\section{Conclusions}

This study revealed interesting clues on how the whole continuum of SBP and DBP were associated with commonly researched factors of hypertension. High-salt diet, drinking and HDL-C were positively associated with BP measures only in males. And the risk of TC had an increased trend with BP in females who used to live in town. 


\begin{tabular}{|c|c|c|c|c|c|c|c|c|c|c|c|c|}
\hline \multirow{2}{*}{\multicolumn{2}{|c|}{ Variables }} & \multicolumn{11}{|l|}{ Quantile } \\
\hline & & \multirow{2}{*}{\begin{tabular}{|l|}
10 \\
$0.15^{*}$ \\
{$[0.11,0.19]$}
\end{tabular}} & \multirow{2}{*}{\begin{tabular}{|l|}
20 \\
$0.23^{*}$ \\
{$[0.19,0.26]$}
\end{tabular}} & \multirow{2}{*}{\begin{tabular}{|l|}
30 \\
$0.27^{*}$ \\
{$[0.24,0.31]$} \\
\end{tabular}} & \multirow{2}{*}{\begin{tabular}{|l|}
40 \\
$0.35^{*}$ \\
{$[0.31,0.38]$}
\end{tabular}} & \multirow{2}{*}{\begin{tabular}{|l|}
$\mathbf{5 0}$ \\
$0.43^{*}$ \\
{$[0.39,0.46]$}
\end{tabular}} & \multirow{2}{*}{\begin{tabular}{|l|}
60 \\
$0.51^{*}$ \\
{$[0.46,0.54]$}
\end{tabular}} & \multirow{2}{*}{\begin{tabular}{|l|}
70 \\
$0.58^{*}$ \\
{$[0.54,0.60]$}
\end{tabular}} & \multirow{2}{*}{$\begin{array}{l}75 \\
0.61^{*} \\
{[0.57,0.65]}\end{array}$} & \multirow{2}{*}{\begin{tabular}{|l|}
80 \\
$0.68^{*}$ \\
{$[0.64,0.73]$}
\end{tabular}} & \multirow{2}{*}{$\begin{array}{l}85 \\
0.73 * \\
{[0.68,0.79]}\end{array}$} & \multirow{2}{*}{$\begin{array}{l}90 \\
0.82^{*} \\
{[0.76,0.88]}\end{array}$} \\
\hline Age (year) & & & & & & & & & & & & \\
\hline BMI $\left(\mathrm{Kg} / \mathrm{m}^{2}\right)$ & & \begin{tabular}{|l|}
$1.15^{*}$ \\
{$[1.00,1.26]$}
\end{tabular} & \begin{tabular}{|l|}
$1.14^{*}$ \\
{$[1.04,1.26]$}
\end{tabular} & \begin{tabular}{|l|}
$1.27^{*}$ \\
{$[1.18,1.40]$}
\end{tabular} & \begin{tabular}{|l|}
$1.32 *$ \\
{$[1.23,1.43]$}
\end{tabular} & \begin{tabular}{|l|}
$1.36^{*}$ \\
{$[1.25,1.46]$}
\end{tabular} & \begin{tabular}{|l|}
$1.43^{*}$ \\
{$[1.28,1.52]$}
\end{tabular} & \begin{tabular}{|l|}
$1.44^{*}$ \\
{$[1.30,1.55]$}
\end{tabular} & $\begin{array}{l}1.51^{*} \\
{[1.36,1.63]}\end{array}$ & $\begin{array}{l}1.53 * \\
{[1.35,1.73]}\end{array}$ & $\begin{array}{l}1.55^{*} \\
{[1.37,1.76]}\end{array}$ & \begin{tabular}{|l|}
$1.66^{*}$ \\
{$[1.46,1.90]$}
\end{tabular} \\
\hline \multirow[b]{2}{*}{ Drinking } & No & - & - & - & - & - & - & - & - & - & - & - \\
\hline & Yes & \begin{tabular}{|l|}
$2.94^{*}$ \\
{$[2.19,3.79]$}
\end{tabular} & \begin{tabular}{|l|}
$3.07^{*}$ \\
{$[2.30,3.81]$}
\end{tabular} & \begin{tabular}{|l|}
$3.10^{*}$ \\
{$[2.48,3.79]$}
\end{tabular} & \begin{tabular}{|l|}
$2.98^{*}$ \\
{$[2.32,3.7]$}
\end{tabular} & \begin{tabular}{|l|}
$3.36^{*}$ \\
{$[2.75,4.17]$}
\end{tabular} & \begin{tabular}{|l|}
$3.7 *$ \\
{$[2.74,4.50]$}
\end{tabular} & \begin{tabular}{|l|}
$3.57 *$ \\
{$[2.51,4.34]$}
\end{tabular} & \begin{tabular}{|l|}
$3.57 *$ \\
{$[2.66,4.49]$}
\end{tabular} & \begin{tabular}{|l|}
$3.74 *$ \\
{$[2.33,4.81]$}
\end{tabular} & $\begin{array}{l}3.75^{*} \\
{[2.50,5.17]}\end{array}$ & \begin{tabular}{|l|}
$4.87^{*}$ \\
{$[3.12,6.16]$}
\end{tabular} \\
\hline \multirow{2}{*}{$\begin{array}{l}\text { High - salt } \\
\text { diet }\end{array}$} & No & - & - & - & - & - & - & - & - & - & - & - \\
\hline & Yes & \begin{tabular}{|l|}
0.22 \\
{$[-0.65,1.09]$}
\end{tabular} & \begin{tabular}{|l|}
0.24 \\
{$[-0.49,0.90]$}
\end{tabular} & \begin{tabular}{|l|}
0.36 \\
{$[-0.32,1.09]$}
\end{tabular} & \begin{tabular}{|l|}
0.15 \\
{$[-0.49,0.88]$}
\end{tabular} & $\begin{array}{l}0.72 \\
{[-0.07,1.44]}\end{array}$ & \begin{tabular}{|l|}
0.74 \\
{$[-0.13,1.68]$}
\end{tabular} & \begin{tabular}{|l|}
0.53 \\
{$[-0.13,1.63]$}
\end{tabular} & $\begin{array}{l}1.10 * \\
{[0.23,1.95]}\end{array}$ & \begin{tabular}{|l|}
$1.47 *$ \\
{$[0.36,2.69]$}
\end{tabular} & $\begin{array}{l}1.45^{*} \\
{[0.19,2.74]}\end{array}$ & \begin{tabular}{|l|}
$2.35^{*}$ \\
{$[0.78,3.97]$}
\end{tabular} \\
\hline \multirow{2}{*}{$\begin{array}{l}\text { Family } \\
\text { history of } \\
\text { CVD }\end{array}$} & No & - & - & - & - & - & - & - & - & - & - & - \\
\hline & Yes & \begin{tabular}{|l|}
$1.20^{*}$ \\
{$[0.42,2.06]$}
\end{tabular} & \begin{tabular}{|l|}
$0.97 *$ \\
{$[0.18,1.65]$}
\end{tabular} & \begin{tabular}{|l|}
$1.23 *$ \\
{$[0.60,1.91]$}
\end{tabular} & \begin{tabular}{|l|}
$1.64^{*}$ \\
{$[0.92,2.32]$}
\end{tabular} & \begin{tabular}{|l|}
$2.08^{*}$ \\
{$[1.25,2.78]$}
\end{tabular} & \begin{tabular}{|l|}
$2.48^{*}$ \\
{$[1.50,3.31]$}
\end{tabular} & \begin{tabular}{|l|}
$2.93 *$ \\
{$[2.01,3.77]$}
\end{tabular} & \begin{tabular}{|l|}
$2.92 *$ \\
{$[2.02,3.68]$}
\end{tabular} & \begin{tabular}{|l|}
$3.12 *$ \\
{$[1.96,4.21]$}
\end{tabular} & $\begin{array}{l}3.12 * \\
{[1.58,4.13]}\end{array}$ & \begin{tabular}{|l|}
$3.69 *$ \\
{$[1.99,5.21]$}
\end{tabular} \\
\hline \multirow{3}{*}{ Education } & Compulsory & - & - & - & - & - & - & - & - & - & - & - \\
\hline & High school & \begin{tabular}{|l|}
0.03 \\
{$[-0.92,1.01]$} \\
\end{tabular} & \begin{tabular}{|l|}
0.49 \\
{$[-0.35,1.63]$}
\end{tabular} & \begin{tabular}{|l|}
0.39 \\
{$[-0.73,1.31]$}
\end{tabular} & \begin{tabular}{|l|}
0.35 \\
{$[-0.72,1.22]$} \\
\end{tabular} & $\begin{array}{l}0.85 \\
{[-0.30,1.66]} \\
\end{array}$ & \begin{tabular}{|l|}
0.55 \\
{$[-0.65,1.58]$}
\end{tabular} & \begin{tabular}{|l|}
0.87 \\
{$[-0.43,1.98]$} \\
\end{tabular} & $\begin{array}{l}0.59 \\
{[-0.62,1.78]}\end{array}$ & $\begin{array}{l}0.22 \\
{[-1.10,2.03]}\end{array}$ & $\begin{array}{l}0.35 \\
{[-1.48,2.34]}\end{array}$ & $\begin{array}{l}-2.28 \\
{[-3.77,0.94]}\end{array}$ \\
\hline & Undergraduate & $\begin{array}{l}-1.11 \\
{[-2.36,0.14]}\end{array}$ & $\begin{array}{l}-0.57 \\
{[-1.37,0.78]}\end{array}$ & $\begin{array}{l}-1.28^{*} \\
{[-2.38} \\
-0.09]\end{array}$ & $\begin{array}{l}-1.27^{*} \\
{[-2.60} \\
-0.04]\end{array}$ & $\begin{array}{l}-1.06^{*} \\
{[-2.56} \\
-0.08]\end{array}$ & $\begin{array}{l}-1.43^{*} \\
{[-2.93} \\
-0.43]\end{array}$ & $\begin{array}{l}-1.93^{*} \\
{[-3.47} \\
-0.53]\end{array}$ & $\begin{array}{l}-2.26^{*} \\
{[-3.46} \\
-0.74]\end{array}$ & $\begin{array}{l}-2.88^{*} \\
{[-4.38} \\
-0.71]\end{array}$ & $\begin{array}{l}-2.63^{*} \\
{[-5.11} \\
-0.32]\end{array}$ & \begin{tabular}{|l}
$-5.26^{*}$ \\
{$[-7.39$} \\
$-2.68]$
\end{tabular} \\
\hline \multirow{2}{*}{ Blood lipid } & TG & \begin{tabular}{|l|}
$0.40^{*}$ \\
{$[0.27,0.69]$} \\
\end{tabular} & \begin{tabular}{|l|}
$0.60 *$ \\
{$[0.34,0.81]$} \\
\end{tabular} & \begin{tabular}{|l|}
$0.72^{*}$ \\
{$[0.51,0.97]$} \\
\end{tabular} & \begin{tabular}{|l|}
$0.79 *$ \\
{$[0.55,0.94]$} \\
\end{tabular} & \begin{tabular}{|l|}
$0.78^{*}$ \\
{$[0.58,1.07]$} \\
\end{tabular} & \begin{tabular}{|l|}
$0.94^{*}$ \\
{$[0.61,1.14]$} \\
\end{tabular} & \begin{tabular}{|l|}
$0.96^{*}$ \\
{$[0.73,1.28]$} \\
\end{tabular} & \begin{tabular}{|l|}
$1.04^{*}$ \\
{$[0.72,1.33]$} \\
\end{tabular} & \begin{tabular}{|l|}
$1.24^{*}$ \\
{$[0.78,1.72]$} \\
\end{tabular} & $\begin{array}{l}1.24^{*} \\
{[0.68,1.74]}\end{array}$ & \begin{tabular}{|l|}
$1.15^{*}$ \\
{$[0.75,1.87]$} \\
\end{tabular} \\
\hline & HDL-C & $\begin{array}{l}2.58^{*} \\
{[0.90,3.98]}\end{array}$ & \begin{tabular}{|l|}
$3.04^{*}$ \\
{$[1.93,3.99]$}
\end{tabular} & \begin{tabular}{|l|}
$3.63^{*}$ \\
{$[2.41,4.62]$}
\end{tabular} & \begin{tabular}{|l|}
$4.00 *$ \\
{$[2.88,4.95]$}
\end{tabular} & \begin{tabular}{|l|}
$3.63^{*}$ \\
{$[2.40,4.63]$}
\end{tabular} & \begin{tabular}{|l|}
$3.96^{*}$ \\
{$[2.76,5.15]$}
\end{tabular} & \begin{tabular}{|l|}
$3.63 *$ \\
{$[2.55,4.98]$}
\end{tabular} & \begin{tabular}{|l|}
$3.78^{*}$ \\
{$[2.41,4.79]$}
\end{tabular} & $\begin{array}{l}3.97 * \\
{[1.68,5.93]}\end{array}$ & $\begin{array}{l}4.25^{*} \\
{[2.57,6.41]}\end{array}$ & $\begin{array}{l}4.69^{*} \\
{[2.45,7.97]}\end{array}$ \\
\hline
\end{tabular}

Table 5. Quantile regression coefficients [ $95 \%$ confidence intervals] of influencing factors for SBP in males. Numbers in the Table are coefficient estimates from the quantile regression with $95 \%$ confidence intervals shown in brackets. ${ }^{*} P<0.05$.

\begin{tabular}{|c|c|c|c|c|c|c|c|c|c|c|c|c|}
\hline \multirow{2}{*}{\multicolumn{2}{|c|}{ Variables }} & \multicolumn{11}{|l|}{ Quantile } \\
\hline & & \multirow{2}{*}{$\begin{array}{l}10 \\
0.07^{*} \\
{[0.04,0.09]}\end{array}$} & \multirow{2}{*}{\begin{tabular}{|l|}
20 \\
$0.10^{*}$ \\
{$[0.07,0.12]$}
\end{tabular}} & \multirow{2}{*}{\begin{tabular}{|l|}
30 \\
$0.12^{*}$ \\
{$[0.10,0.14]$}
\end{tabular}} & \multirow{2}{*}{$\begin{array}{l}40 \\
0.15^{*} \\
{[0.13,0.17]}\end{array}$} & \multirow{2}{*}{\begin{tabular}{|l|}
$\mathbf{5 0}$ \\
$0.16^{*}$ \\
{$[0.14,0.18]$}
\end{tabular}} & \multirow{2}{*}{\begin{tabular}{|l|}
60 \\
$0.18^{*}$ \\
{$[0.16,0.21]$}
\end{tabular}} & \multirow{2}{*}{\begin{tabular}{|l|}
70 \\
$0.20^{*}$ \\
{$[0.18,0.23]$}
\end{tabular}} & \multirow{2}{*}{\begin{tabular}{|l|}
75 \\
$0.23 *$ \\
{$[0.20,0.25]$}
\end{tabular}} & \multirow{2}{*}{\begin{tabular}{|l|}
$\mathbf{8 0}$ \\
$0.22 *$ \\
{$[0.20,0.27]$}
\end{tabular}} & \multirow{2}{*}{\begin{tabular}{|l}
85 \\
$0.25^{*}$ \\
{$[0.22,0.29]$}
\end{tabular}} & \multirow{2}{*}{\begin{tabular}{|l|}
90 \\
$0.27^{*}$ \\
{$[0.22,0.31]$}
\end{tabular}} \\
\hline Age (year) & & & & & & & & & & & & \\
\hline $\begin{array}{l}\mathrm{BMI}(\mathrm{Kg} / \\
\left.\mathrm{m}^{2}\right)\end{array}$ & & $\begin{array}{l}0.67 * \\
{[0.57,0.76]}\end{array}$ & $\begin{array}{l}0.68^{*} \\
{[0.60,0.75]}\end{array}$ & $\begin{array}{l}0.78^{*} \\
{[0.70,0.86]}\end{array}$ & \begin{tabular}{|l|}
$0.85^{*}$ \\
{$[0.78,0.92]$}
\end{tabular} & \begin{tabular}{|l}
$0.88^{*}$ \\
{$[0.82,0.96]$}
\end{tabular} & $\begin{array}{l}0.91 * \\
{[0.84,0.98]}\end{array}$ & $\begin{array}{l}0.92 * \\
{[0.86,1.02]}\end{array}$ & $\begin{array}{l}0.91 * \\
{[0.83,1.02]}\end{array}$ & $\begin{array}{l}0.95^{*} \\
{[0.84,1.03]}\end{array}$ & $\begin{array}{l}0.98^{*} \\
{[0.87,1.08]}\end{array}$ & $\begin{array}{l}1.03 * \\
{[0.87,1.17]}\end{array}$ \\
\hline \multirow[b]{2}{*}{ Drinking } & No & - & - & - & - & - & - & - & - & - & - & - \\
\hline & Yes & $\begin{array}{l}2.29 * \\
{[1.69,2.85]}\end{array}$ & \begin{tabular}{|l|}
$2.1 *$ \\
{$[1.47,2.59]$} \\
\end{tabular} & $\begin{array}{l}2.15^{*} \\
{[1.74,2.72]} \\
\end{array}$ & \begin{tabular}{|l|}
$2.34^{*}$ \\
{$[1.91,2.77]$} \\
\end{tabular} & \begin{tabular}{|l|}
$2.57 *$ \\
{$[2.01,3.03]$} \\
\end{tabular} & \begin{tabular}{|l|}
$2.90^{*}$ \\
{$[2.44,3.42]$} \\
\end{tabular} & \begin{tabular}{|l|}
$3.11^{*}$ \\
{$[2.47,3.75]$} \\
\end{tabular} & \begin{tabular}{|l|}
$2.94 *$ \\
{$[2.34,3.67]$} \\
\end{tabular} & \begin{tabular}{|l|}
$2.84^{*}$ \\
{$[2.22,3.57]$} \\
\end{tabular} & $\begin{array}{l}3.16^{*} \\
{[2.22,3.89]} \\
\end{array}$ & \begin{tabular}{|l|}
$2.72 *$ \\
{$[1.84,3.41]$} \\
\end{tabular} \\
\hline \multirow{2}{*}{$\begin{array}{l}\text { High - salt } \\
\text { diet }\end{array}$} & No & - & - & - & - & - & - & - & - & - & - & - \\
\hline & Yes & $\begin{array}{l}0.80^{*} \\
{[0.19,1.38]}\end{array}$ & $\begin{array}{l}0.41 \\
{[-0.04,1.00]}\end{array}$ & \begin{tabular}{|l|}
$0.71 *$ \\
{$[0.23,1.25]$} \\
\end{tabular} & \begin{tabular}{|l|}
$0.82^{*}$ \\
{$[0.38,1.22]$} \\
\end{tabular} & \begin{tabular}{|l|}
$0.80^{*}$ \\
{$[0.27,1.20]$} \\
\end{tabular} & \begin{tabular}{|l|}
$0.87^{*}$ \\
{$[0.43,1.36]$} \\
\end{tabular} & $\begin{array}{l}0.89^{*} \\
{[0.35,1.43]} \\
\end{array}$ & \begin{tabular}{|l|}
$0.83^{*}$ \\
{$[0.19,1.39]$} \\
\end{tabular} & \begin{tabular}{|l|}
$0.70 *$ \\
{$[0.06,1.32]$} \\
\end{tabular} & $\begin{array}{l}0.56 \\
{[-0.05,1.43]} \\
\end{array}$ & \begin{tabular}{|l|}
0.72 \\
{$[-0.10,1.86$} \\
\end{tabular} \\
\hline \multirow{2}{*}{$\begin{array}{l}\text { Family } \\
\text { history of } \\
\text { CVD }\end{array}$} & No & - & - & - & - & - & - & - & - & - & - & - \\
\hline & Yes & $\begin{array}{l}0.58^{*} \\
{[0.06,1.29]}\end{array}$ & $\begin{array}{l}0.97 * \\
{[0.51,1.51]}\end{array}$ & $\begin{array}{l}1.16^{*} \\
{[0.67,1.65]}\end{array}$ & $\begin{array}{l}1.37 * \\
{[0.92,1.85]}\end{array}$ & \begin{tabular}{|l}
$1.76^{*}$ \\
{$[1.31,2.23]$}
\end{tabular} & $\begin{array}{l}.04^{*} \\
{[1.50,2.53]}\end{array}$ & $\begin{array}{l}2.30 * \\
{[1.74,2.87]}\end{array}$ & $\begin{array}{l}2.68 * \\
{[2.02,3.22]}\end{array}$ & $\begin{array}{l}2.63 * \\
{[2.00,3.29]}\end{array}$ & $\begin{array}{l}2.52 * \\
{[1.82,3.28]}\end{array}$ & \begin{tabular}{|l|}
$2.81 *$ \\
{$[2.09,3.64]$}
\end{tabular} \\
\hline \multirow{3}{*}{ Occupation } & Other* & - & - & - & - & - & - & - & - & - & - & - \\
\hline & $\begin{array}{l}\text { Manual } \\
\text { labor }\end{array}$ & $\begin{array}{l}1.77^{*} \\
{[0.71,3.17]}\end{array}$ & $\begin{array}{l}1.51^{*} \\
{[0.46,2.12]} \\
\end{array}$ & $\begin{array}{l}1.40 * \\
{[0.71,2.05]}\end{array}$ & \begin{tabular}{|l|}
$1.54^{*}$ \\
{$[0.81,2.00]$} \\
\end{tabular} & $\begin{array}{l}1.32 * \\
{[0.46,2.13]}\end{array}$ & $\begin{array}{l}1.39 * \\
{[0.55,2.33]}\end{array}$ & $\begin{array}{l}1.06^{*} \\
{[0.26,1.82]}\end{array}$ & $\begin{array}{l}1.39 * \\
{[0.26,2.08]}\end{array}$ & $\begin{array}{l}1.22 * \\
{[0.38,2.20]} \\
\end{array}$ & $\begin{array}{l}1.36^{*} \\
{[0.36,2.20]}\end{array}$ & \begin{tabular}{|l|}
$1.91 *$ \\
{$[1.15,3.03]$} \\
\end{tabular} \\
\hline & $\begin{array}{l}\text { Mental } \\
\text { labor }\end{array}$ & $\begin{array}{l}2.03 * \\
{[1.32,3.36]}\end{array}$ & $\begin{array}{l}2.21^{*} \\
{[1.05,3.04]} \\
\end{array}$ & $\begin{array}{l}2.36^{*} \\
{[1.56,3.00]}\end{array}$ & \begin{tabular}{|l|}
$2.47^{*}$ \\
{$[1.59,3.02]$} \\
\end{tabular} & \begin{tabular}{|l|}
$2.18^{*}$ \\
{$[1.24,3.02]$} \\
\end{tabular} & \begin{tabular}{|l|}
$2.01 *$ \\
{$[1.15,2.95]$} \\
\end{tabular} & \begin{tabular}{|l|}
$1.52 *$ \\
{$[0.73,2.36]$} \\
\end{tabular} & $\begin{array}{l}1.79 * \\
{[0.55,2.57]}\end{array}$ & \begin{tabular}{|l|}
$1.45^{*}$ \\
{$[0.63,2.58]$} \\
\end{tabular} & $\begin{array}{l}1.51^{*} \\
{[0.46,2.48]} \\
\end{array}$ & \begin{tabular}{|l|}
$2.42 *$ \\
{$[1.31,3.92]$} \\
\end{tabular} \\
\hline \multirow{2}{*}{ Blood lipid } & TG & $\begin{array}{l}0.50 * \\
{[0.43,0.70]}\end{array}$ & \begin{tabular}{|l|}
$0.76^{*}$ \\
{$[0.54,0.87]$} \\
\end{tabular} & $\begin{array}{l}0.74^{*} \\
{[0.61,0.83]} \\
\end{array}$ & $\begin{array}{l}0.75^{*} \\
{[0.66,0.89]} \\
\end{array}$ & \begin{tabular}{|l|}
$0.76^{*}$ \\
{$[0.65,0.93]$} \\
\end{tabular} & \begin{tabular}{|l|}
$0.78^{*}$ \\
{$[0.64,0.96]$} \\
\end{tabular} & \begin{tabular}{|l|}
$0.79^{*}$ \\
{$[0.61,1.02]$} \\
\end{tabular} & \begin{tabular}{|l|}
$0.87 *$ \\
{$[0.60,1.05]$} \\
\end{tabular} & \begin{tabular}{|l|}
$0.85 *$ \\
{$[0.62,1.10]$} \\
\end{tabular} & \begin{tabular}{|l|}
$0.87 *$ \\
{$[0.67,1.29]$} \\
\end{tabular} & \begin{tabular}{|l|}
$1.25^{*}$ \\
{$[0.77,1.71]$} \\
\end{tabular} \\
\hline & HDL-C & $\begin{array}{l}0.92 * \\
{[0.17,1.38]}\end{array}$ & $\begin{array}{l}1.85^{*} \\
{[0.84,2.52]}\end{array}$ & $\begin{array}{l}2.41^{*} \\
{[1.67,2.85]}\end{array}$ & $\begin{array}{l}2.26^{*} \\
{[1.75,3.08]}\end{array}$ & $\begin{array}{l}2.65^{*} \\
{[1.92,3.47]}\end{array}$ & $\begin{array}{l}2.78^{*} \\
{[1.92,3.40]}\end{array}$ & \begin{tabular}{|l|}
$2.57^{*}$ \\
{$[1.68,3.71]$}
\end{tabular} & $\begin{array}{l}2.63 * \\
{[1.89,3.43]}\end{array}$ & \begin{tabular}{|l}
$.97 *$ \\
{$[1.94,4.01]$}
\end{tabular} & \begin{tabular}{|l|}
$3.00 *$ \\
{$[2.19,4.86]$}
\end{tabular} & \begin{tabular}{|l|}
$4.37 *$ \\
{$[2.84,5.77]$}
\end{tabular} \\
\hline
\end{tabular}

Table 6. Quantile regression coefficients [ $95 \%$ confidence intervals] of influencing factors for DBP in males. Numbers in the Table are coefficient estimates from the quantile regression with $95 \%$ confidence intervals shown in brackets. ${ }^{*} P<0.05$, "other" included unemployed and retired people.

The elderly, the obese, workers and people with lower level of education and family history of CVD were expected to be positively associated with a higher SBP and/or DBP.

\section{Methods}

Study population. The large-scale cross-sectional survey was conducted among people who aged 18 to79 years old and were living in Jilin Province for over 6 months in 2012. A total of 23,050 participants were selected through multistage stratified random cluster sampling ${ }^{32}$ (see details in Part 1 of the Supplementary Material). For the purpose of the present analysis, the subjects who had anti-hypertensive treatments were excluded, as well as 


\begin{tabular}{|c|c|c|c|c|c|c|c|c|c|c|}
\hline \multirow[b]{2}{*}{ Quantiles } & \multicolumn{2}{|l|}{ Total } & \multicolumn{2}{|c|}{ Underweight } & \multicolumn{2}{|l|}{ Normal } & \multicolumn{2}{|c|}{ Overweight } & \multicolumn{2}{|l|}{ Obese } \\
\hline & Normal & Hypertension & Normal & Hypertension & Normal & Hypertension & Normal & Hypertension & Normal & Hypertension \\
\hline 10 & 0.92 & 0.88 & 1.01 & 0.98 & 1.11 & 1.13 & 0.87 & 0.88 & 0.80 & 0.82 \\
\hline 20 & 1.04 & 0.99 & 1.14 & 1.13 & 1.30 & 1.29 & 0.97 & 0.98 & 0.90 & 0.90 \\
\hline 30 & 1.13 & 1.09 & 1.23 & 1.24 & 1.47 & 1.36 & 1.05 & 1.06 & 0.97 & 0.98 \\
\hline 40 & 1.22 & 1.17 & 1.32 & 1.33 & 1.67 & 1.46 & 1.11 & 1.15 & 1.03 & 1.04 \\
\hline 50 & 1.30 & 1.26 & 1.40 & 1.44 & 1.78 & 1.53 & 1.19 & 1.22 & 1.09 & 1.11 \\
\hline 60 & 1.39 & 1.35 & 1.49 & 1.55 & 1.90 & 1.63 & 1.26 & 1.31 & 1.15 & 1.18 \\
\hline 70 & 1.50 & 1.46 & 1.61 & 1.68 & 1.97 & 1.72 & 1.35 & 1.41 & 1.22 & 1.25 \\
\hline 75 & 1.57 & 1.54 & 1.68 & 1.75 & 2.13 & 1.79 & 1.40 & 1.46 & 1.26 & 1.30 \\
\hline 80 & 1.64 & 1.62 & 1.75 & 1.85 & 2.23 & 1.92 & 1.47 & 1.53 & 1.30 & 1.36 \\
\hline 85 & 1.74 & 1.72 & 1.85 & 1.96 & 2.33 & 2.03 & 1.54 & \begin{tabular}{|l|l}
1.60 \\
\end{tabular} & \begin{tabular}{|l|l}
1.35 \\
\end{tabular} & \begin{tabular}{|l|l|}
1.43 \\
\end{tabular} \\
\hline 90 & \begin{tabular}{|l|}
1.87 \\
\end{tabular} & \begin{tabular}{|l|}
1.86 \\
\end{tabular} & \begin{tabular}{|l|}
1.98 \\
\end{tabular} & 2.13 & 2.58 & \begin{tabular}{|l|}
2.26 \\
\end{tabular} & \begin{tabular}{|l|}
1.67 \\
\end{tabular} & \begin{tabular}{|l|}
1.71 \\
\end{tabular} & \begin{tabular}{|l|}
1.42 \\
\end{tabular} & \begin{tabular}{|l|}
1.50 \\
\end{tabular} \\
\hline
\end{tabular}

Table 7. Quantiles of HDL-C for different BMI groups in males (mmol/L). Underweight: $\mathrm{BMI}<18.5 \mathrm{~kg} / \mathrm{m}^{2}$, Normal: $18.5 \mathrm{~kg} / \mathrm{m}^{2} \leq \mathrm{BMI}<24.0 \mathrm{~kg} / \mathrm{m}^{2}$, Overweight: $24.0 \mathrm{~kg} / \mathrm{m}^{2} \leq \mathrm{BMI}<28.0 \mathrm{~kg} / \mathrm{m}^{2}$, Obese: BMI $\geq 28.0 \mathrm{~kg} / \mathrm{m}$.

SBP

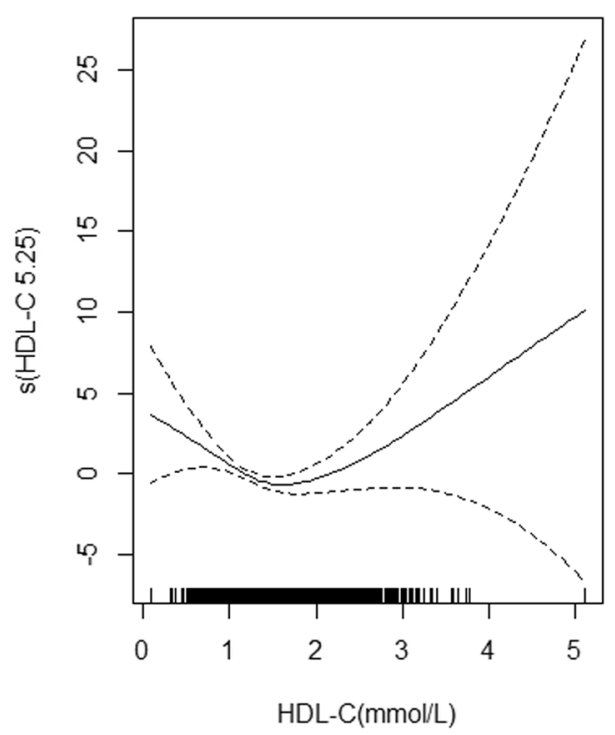

DBP

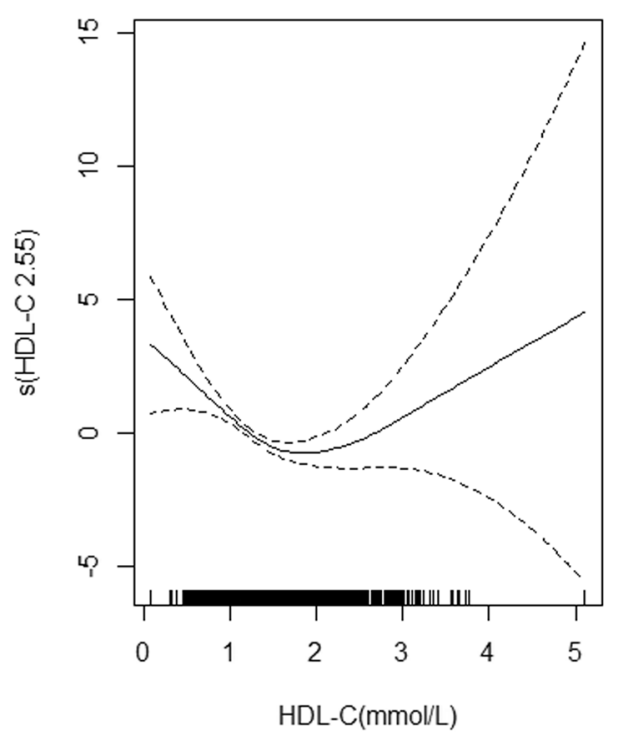

Figure 1. The association of BP and HDL-C in Generalized Additive Models (GAM). The vertical axis represents the smoothness function value, the numbers in brackets represent the estimated degrees of freedom (EDF), and the dotted line represents the lower limit of the confidence interval.

those who had missing values in SBP or DBP. Finally, a total of 16,524 subjects were included in the present analyses. All participants provided written informed consent, and the study was approved by the Institutional Review Board of the School of Public Health, Jilin University. And all methods were performed in accordance with the relevant guidelines and regulations.

Data collection and measurement. The data of this study included demographics (e.g., gender, age, etc.), health-related behaviors (e.g., smoking, drinking, etc.), anthropometric measurements (e.g., height, weight, hypertension, etc.) and laboratory measurements (such as serum cholesterol and triglycerides). All investigation was trained and followed the same questionnaire instructions.

Height and weight were measured according to a standardized protocol and techniques, with the participants wearing clothing but no shoes. A calibrated mercury sphygmomanometer was used to determine the blood pressure of subjects on the right arm, after at least $5 \mathrm{~min}$ of seated rest. The blood sample was obtained in the morning from subjects after fasting for at least eight hours, and then conserved in tubes which contained ethylene diamine tetra acetic acid (EDTA). Fasting plasma glucose (FPG) and serum lipids were measured using a Bai Ankang fingertip blood glucose monitor (Bayer, Leverkusen, Germany) and a MODULE P800 biochemical analysis machine (Roche Co., Ltd., Shanghai, China), respectively ${ }^{33}$ (see details in Part 2 of the Supplementary Material).

Assessment criteria. According to Seventh Joint National Commission Guidelines (JNC7), hypertension was defined as a resting systolic blood pressure (SBP)/diastolic blood pressure (DBP) $\geq 140 / 90 \mathrm{mmHg}$ or current 
use of antihypertensive medication ${ }^{34}$. Dyslipidemia was defined as the use of lipid-lowering drugs in the past two weeks and/or meeting one or more of the following criteria: total cholesterol (TC) $\geq 6.22 \mathrm{mmol} / \mathrm{L}$, triglyceride $(\mathrm{TG}) \geq 2.26 \mathrm{mmol} / \mathrm{L}$, low density lipoprotein cholesterol (LDL-C) $\geq 4.14 \mathrm{mmol} / \mathrm{L}$, high density lipoprotein cholesterol $(\mathrm{HDL}-\mathrm{C})<1.04 \mathrm{mmol} / \mathrm{L}^{35}$. We defined diabetes as participants who reported diabetes mellitus previously diagnosed by physician or those who have fasting plasma glucose (FPG) $\geq 7.0 \mathrm{mmol} / \mathrm{L}$ or oral glucose tolerance test (OGTT) $2 \mathrm{~h}$ plasma glucose $(\mathrm{PG}) \geq 11.1 \mathrm{mmol} / \mathrm{L}^{36}$. BMI was defined as weight (kilogram) divided by height (meter) squared. Drinker was defined as a person who consumed more than one alcoholic drink per week, including any form of alcohol ${ }^{37}$. High-salt diet was defined as a person who daily salt intake more than $6 \mathrm{~g}^{38}$. Education stage was classified into compulsory education and lower education, secondary school education, senior school education, college education, postgraduate education and higher education.

Statistical Analysis. All questionnaires were coded and double-entered. EpiData (version 3.1) was used for data entry and validation and R version 3.3.3(University of Auckland, Oakland, New Zealand) for data analysis. The data are presented as mean \pm standard deviations (SD) or rate. In addition, quantitative variables and categorical variables were analyzed using $t$ test and Rao-scott- $\chi 2$ test, respectively. Finally, QR in the quantreg package was used to estimate the conditional quantile of the distribution of SBP and DBP under the influence of various risk factors.

Data Availability. The survey was implemented by School of Public Health, Jilin University and Jilin Center for Disease Control and Prevention in Jilin Province in 2012. According to relevant regulations, we were sorry that the data can't be shared.

About the data. The survey was implemented by School of Public Health, Jilin University and Jilin Center for Disease Control and Prevention in Jilin Province in 2012. According to relevant regulations, we were sorry that the data can't be shared.

\section{References}

1. Yu, J., Ma, Y., Yang, S., Pang, K., Yu, Y. \& Tao, Y. et al. Risk Factors for Cardiovascular Disease and Their Clustering among Adults in Jilin (China). Int J Environ Res Public Health 13, h13010070 (2015).

2. Yu, D., Huang, J., Hu, D., Chen, J., Cao, J. \& Li, J. et al. Association between prehypertension and clustering of cardiovascular disease risk factors among Chinese adults. J Cardiovasc Pharmacol 53, 388-400 (2009).

3. Unal, S., Acar, B., Ertem, A. G. \& Sen, F. Endocan in Hypertension and Cardiovascular Diseases. ANGIOLOGY 68, 85 (2017).

4. Lu, X., Huang, J., Wang, L., Chen, S., Yang, X. \& Li, J. et al. Genetic predisposition to higher blood pressure increases risk of incident hypertension and cardiovascular diseases in Chinese. HYPERTENSION 66, 786-792 (2015).

5. Liu, X., Gu, W., Li, Z., Lei, H., Li, G. \& Huang, W. Hypertension prevalence, awareness, treatment, control, and associated factors in Southwest China: an update. J HYPERTENS 35, 637-644 (2017).

6. Ma, W. J. et al. Hypertension prevalence, awareness, treatment, control, and associated factors in adults in southern China. AM J HYPERTENS 25, 590-596 (2012).

7. Gao, Y., Chen, G., Tian, H., Lin, L., Lu, J. \& Weng, J. et al. Prevalence of hypertension in china: a cross-sectional study. PLOS ONE 8 , e65938 (2013).

8. Shen, X., Li, K., Chen, P., Feng, R., Liang, H. \& Tong, G. et al. Associations of Blood Pressure with Common Factors among LeftBehind Farmers in Rural China. MEDICINE 94, e142 (2015).

9. Aounallah-Skhiri, H. et al. Blood pressure and associated factors in a North African adolescent population. A national crosssectional study in Tunisia. BMC Public Health 12, 98 (2012).

10. Sun, H. et al. Waist circumference and incidence of hypertension in Chinese adults: Observations from the Kailuan Study. HERZ (2016).

11. Diederichs, C. \& Neuhauser, H. The incidence of hypertension and its risk factors in the German adult population: results from the German National Health Interview and Examination Survey 1998 and the German Health Interview and Examination Survey for Adults 2008-2011. J HYPERTENS 35, 250-258 (2017).

12. Rautiainen, S. et al. Multivitamin use and the risk of hypertension in a prospective cohort study of women. J HYPERTENS 34, 1513-1519 (2016).

13. Zhang $\mathrm{M}$ et al. Effect of dynamic change in body mass index on the risk of hypertension: Results from the Rural Chinese Cohort Study. INT J CARDIOL (2017).

14. Wei, Y., Pere, A., Koenker, R. \& He, X. Quantile regression methods for reference growth charts. STAT MED 25, 1369-1382 (2006).

15. Wei, Q. et al. Prevalence of hypertension and associated risk factors in Dehui City of Jilin Province in China. J HUM HYPERTENS 29, 64-68 (2015).

16. Olack, B. et al. Risk factors of hypertension among adults aged 35-64 years living in an urban slum Nairobi, Kenya. BMC PUBLIC HEALTH 15, 1251 (2015).

17. Wakabayashi, I. Influence of gender on the association of alcohol drinking with blood pressure. AM J HYPERTENS 21, 1310-1317 (2008).

18. Krupp, D., Shi, L., Egert, S., Wudy, S. A. \& Remer, T. Prospective relevance of fruit and vegetable consumption and salt intake during adolescence for blood pressure in young adulthood. EUR J NUTR 54, 1269-1279 (2015).

19. Kim, J. M., Kim, T. H., Lee, H. H., Lee, S. H. \& Wang, T. Postmenopausal hypertension and sodium sensitivity. J Menopausal Med 20, $1-6(2014)$.

20. Czernichow, S., Castetbon, K., Salanave, B., Vernay, M., Barry, Y. \& Batty, G. D. et al. Determinants of blood pressure treatment and control in obese people: evidence from the general population. J HYPERTENS 30, 2338-2344 (2012).

21. Hall, J. E., Brands, M. W., Hildebrandt, D. A., Kuo, J. \& Fitzgerald, S. Role of sympathetic nervous system and neuropeptides in obesity hypertension. BRAZ J MED BIOL RES 33(6), 605-618 (2000)

22. Bonaa, K. H. \& Thelle, D. S. Association between blood pressure and serum lipids in a population. The Tromso Study. CIRCULATION 83, 1305-1314 (1991).

23. Tangvarasittichai, S. Oxidative stress, insulin resistance, dyslipidemia and type 2 diabetes mellitus. World J Diabetes 6, 456-480 (2015).

24. Chavez-Canales, M., Arroyo, J. P., Ko, B., Vazquez, N., Bautista, R. \& Castaneda-Bueno, M. et al. Insulin increases the functional activity of the renal $\mathrm{NaCl}$ cotransporter. J HYPERTENS 31, 303-311 (2013).

25. Onat, A., Karadeniz, Y., Tusun, E., Yuksel, H. \& Kaya, A. Advances in understanding gender difference in cardiometabolic disease risk. Expert Rev Cardiovasc Ther 14, 513-523 (2016). 
26. Wakabayashi, I. Associations of Blood Lipid-Related Indices with Blood Pressure and Pulse Pressure in Middle-Aged Men. METAB SYNDR RELAT D 13, 22-28 (2015).

27. Carroll, D., Phillips, A. C., Gale, C. R. \& Batty, G. D. Generalized anxiety and major depressive disorders, their comorbidity and hypertension in middle-aged men. PSYCHOSOM MED 72, 16-19 (2010).

28. Fan, W., Qu, X., Li, J., Wang, X., Bai, Y. \& Cao, Q. et al. Associations between polymorphisms of the ADIPOQ gene and hypertension risk: a systematic and meta-analysis. Sci Rep 7, 41683 (2017).

29. Gupta, R. et al. Association of Household Wealth Index, Educational Status, and Social Capital with Hypertension Awareness, Treatment, and Control in South Asia. AM J HYPERTENS (2017).

30. Franklin, S. S. et al. Hemodynamic patterns of age-related changes in blood pressure. The Framingham Heart Study. CIRCULATION 96, 308-315 (1997).

31. Williamson, C., Jeemon, P., Hastie, C. E., McCallum, L., Muir, S. \& Dawson, J. et al. Family history of premature cardiovascular disease: blood pressure control and long-term mortality outcomes in hypertensive patients. EUR HEART J 35, 563-570 (2014).

32. Wang, C., Yu, Y., Zhang, X., Li, Y., Kou, C. \& Li, B. et al. Awareness, treatment, control of diabetes mellitus and the risk factors: survey results from northeast China. PLOS ONE 9, e103594 (2014).

33. Xie, X., Ma, Y. T., Yang, Y. N., Fu, Z. Y., Li, X. M. \& Zheng, Y. Y. et al. Polymorphisms in the SAA1 gene are associated with ankle-tobrachial index in Han Chinese healthy subjects. Blood Press 20, 232-238 (2011).

34. Executive Summary of the Third Report of the National Cholesterol Education Program (NCEP). Expert Panel on Detection, Evaluation, and Treatment of High Blood Cholesterol in Adults (Adult Treatment Panel III). JAMA 285, 2486-2497 (2001).

35. Dong, X., Liu, Y., Yang, J., Sun, Y. \& Chen, L. Efficiency of anthropometric indicators of obesity for identifying cardiovascular risk factors in a Chinese population. POSTGRAD MED J 87, 251-256 (2011).

36. Basevi V, Di Mario S, Morciano C, Nonino F \& Magrini N. Comment on: American Diabetes Association. Standards of medical care in diabetes-2011. Diabetes Care 201134(Suppl. 1):S11-S61. DIABETES CARE 34, e53, e54 (2011).

37. Zhang P, Wang R, Gao C, Song Y, Lv X \& Jiang L et al. Types of Obesity and Its Association with the Clustering of Cardiovascular Disease Risk Factors in Jilin Province of China. Int J Environ Res Public Health 13, (2016).

38. Lewington, S., Clarke, R., Qizilbash, N., Peto, R. \& Collins, R. Age-specific relevance of usual blood pressure to vascular mortality: a meta-analysis of individual data for one million adults in 61 prospective studies. LANCET 360, 1903-1913 (2002).

\section{Acknowledgements}

I would like to express my gratitude to all those who have helped me during the writing of this thesis. This work was supported by the Outstanding Youth Foundation of Science and Technology Department of Jilin Province, China (grant number: 20170520049JH); the National Natural Science Foundation of China (grant number: 11301213, 11571068); and the Scientific Research Foundation of the Health Bureau of Jilin Province, China (grant number: 2011Z116).

\section{Author Contributions}

The authors would like to thank Dr Lina Jin and others who kindly provided their R code for analysis and experience for writing paper. Junsen Ye and Lina Jin had the original idea for the study and carried out the design. Lina Jin and Zhongmin Li provided valuable insight regarding the methodological approach and organization of the manuscript. Junsen Ye, Jianxing Yu, Yaogai Lv and Lan An carried out the statistical analysis and reviewed the consistency of data included in the paper. Junsen Ye and Zhongmin Li drafted the manuscript. Xin Guo, Yan Yao and Yaqin Yu revised the manuscript. All authors read and approved the final manuscript.

\section{Additional Information}

Supplementary information accompanies this paper at https://doi.org/10.1038/s41598-017-14045-0.

Competing Interests: The authors declare that they have no competing interests.

Publisher's note: Springer Nature remains neutral with regard to jurisdictional claims in published maps and institutional affiliations.

(c) (i) Open Access This article is licensed under a Creative Commons Attribution 4.0 International

License, which permits use, sharing, adaptation, distribution and reproduction in any medium or format, as long as you give appropriate credit to the original author(s) and the source, provide a link to the Creative Commons license, and indicate if changes were made. The images or other third party material in this article are included in the article's Creative Commons license, unless indicated otherwise in a credit line to the material. If material is not included in the article's Creative Commons license and your intended use is not permitted by statutory regulation or exceeds the permitted use, you will need to obtain permission directly from the copyright holder. To view a copy of this license, visit http://creativecommons.org/licenses/by/4.0/.

(C) The Author(s) 2017 